\title{
Förderung der Textkompetenz anhand der Textsorte Arbeitszeugnis
}

\section{Tomáš Káňa und Justina Kirchner}

The article describes the structure and several formulations of one of the typical German text types Arbeitszeugnis (Reference paper from the employer for the employee) and shows how to implement this text type into German language teaching focussing on enhancing the discourse competence. The description of this text type includes legal frames in Austria, Germany and Czechia. The implementation into teaching follows SchmölzerEibinger's 3phase model for promoting text competence and demonstrates how to deal with such texts, which seem to be quite complicated even for native speakers. The Arbeitszeugnis should be a topic in German language teaching for its specific features and because it is an integral part of vocational life in German speaking countries.

discourse competence - text competence - promoting text competence - 3-phase-model text type - reference paper

Der Artikel beschreibt die Struktur und einige Formulierungen der Textsorte Arbeitszeugnis und zeigt, wie diese Textsorte im Deutschunterricht eingesetzt werden kann, um die Textkompetenz zu fördern. Die Beschreibung der Textsorte enthält auch rechtliche Rahmenbedingungen für die Erstellung von Arbeitszeugnissen in Österreich, Deutschland und in Tschechien. Die Umsetzung im Unterricht folgt Schmölzer-Eibingers 3-Phasen-Modell zur Förderung der Textkompetenz und zeigt, wie man mit Texten umgehen kann, die selbst für Muttersprachler/-innen relativ kompliziert sind. Die Textsorte Arbeitszeugnis sollte ein Thema im Deutschunterricht sein, weil es sich um eine spezifische Textsorte handelt und sie ein fester Bestandteil des Berufslebens im deutschsprachigen Raum ist.

Textkompetenz - Förderung - 3-Phasen-Modell - Textsorte - Arbeitszeugnis

\section{Einleitung}

Texte begleiten Fremdsprachenlernende vom Anfang ihres Lernprozesses an. Sie sind „Ausgangspunkt und Ziel“ des Unterrichts (Meier 2006: 193). Die Textrezeption und produktion stellt allerdings nicht nur für eine große Zahl der Lernenden des Deutschen als Fremd- oder Zweitsprache, sondern auch für Muttersprachler/-innen (L-1-Lernende) eine große Schwierigkeit dar.

Der Ansatz der Textkompetenz und der Forschungen in diesem Bereich sind deshalb über die letzten Jahre hinweg immer mehr ins Blickfeld gerückt. Da die Arbeit mit und an Texten im Unterricht einen so zentralen Stellenwert einnimmt, ist die Fähigkeit, Texte lesen, verstehen, kommentieren und produzieren zu können, ein Schlüsselelement zum Erfolg in Bildungsinstitutionen. Entsprechend findet man mittlerweile verschiedenste Ansätze und Modelle, die die Förderung der Textkompetenz oder jeweils der Lese- und Schreibkompetenz zum Ziel haben. Doch Texte werden nicht nur im (Fremdsprachen- oder Fach-) Unterricht gelesen und 
produziert. Sie sind in Form verschiedener Textsorten auch im Alltag ständig präsent und haben unterschiedlich große Bedeutung für uns Sprachbenutzer/-innen. Eine dieser Textsorten ist das Arbeitszeugnis, das für alle, die im deutschsprachigen Raum einer Arbeit nachgehen oder sich um eine Stelle bewerben, relevant ist, da im Rahmen des Bewerbungsprozesses dem Arbeitszeugnis große Bedeutung zukommt. Auch unerfahrenen Muttersprachlerinnen und Muttersprachlern fallen mehrere Besonderheiten dieser Textsorte auf - vor allem die "Geheimsprache“, die sie auszeichnet. Auf Nicht-Muttersprachler/-innen des Deutschen dürfte diese Textsorte noch kryptischer wirken, denn in vielen Staaten existiert die Textsorte in dieser Form ${ }^{1}$ nicht. Die tschechische Arbeitswelt etwa kennt keine direkte Entsprechung. Am häufigsten wird Arbeitszeugnis als potvrzeni o zamèstnáni bzw. zápočtový list übersetzt. Beides entspricht jedoch bloß einer Arbeitsbestätigung.

In diesem Kontext soll nun der Frage nachgegangen werden, ob und inwiefern sich die Textsorte Arbeitszeugnis für den Einsatz im DaF- oder DaZ-Unterricht eignet und wie von ihr ausgehende Fördermöglichkeiten zur Textkompetenz aussehen könnten. Hierfür soll in Kapitel 2 der recht breite Begriff der Textkompetenz eingegrenzt und für den Zweck der Beantwortung obenstehender Fragestellung umrissen werden. Danach wird darauf eingegangen, wie die Textkompetenz gefördert werden kann, wobei ein genauerer Blick auf das 3-Phasen-Modell von SchmölzerEibinger (2011) geworfen wird.

In Kapitel 4 wird die Textsorte Arbeitszeugnis genauer vorgestellt und ihre wichtigsten strukturellen und sprachlichen Merkmale sowie ihre Besonderheiten beschrieben.

$\mathrm{Ob}$ und inwiefern sich das Arbeitszeugnis für den Einsatz im DaF- und DaZUnterricht eignet und welche Textmerkmale und sprachlichen Mittel sich im Speziellen für eine Thematisierung und Bearbeitung mit Lernenden anbieten, wird in Kapitel 5 diskutiert. Dazu werden auf die Textsorte abgestimmte Fördermaßnahmen und Aufgaben präsentiert und beschrieben.

Schließlich fassen wir im letzten Kapitel die Erkenntnisse zusammen und bringen ein Fazit zum Einsatz der Textsorte Arbeitszeugnis im Unterricht sowie zu ihrer Eignung für die Förderung der Textkompetenz.

\section{Begriffsbestimmung}

In der heutigen Welt geschieht die Übermittlung und Verbreitung von Wissen größtenteils in Form von Texten. Dies gilt auch für den Unterricht, ganz besonders auch für den Fremdsprachenunterricht, in dem der Text an sich die „zentrale

1 Die Charakteristik von Duden (online) „einem Arbeitnehmer, einer Arbeitnehmerin vom Arbeitgeber ausgestelltes schriftliches Zeugnis" (14.3.2018) darf nicht in die Irre führen, dass es sich um eine „Benotung“ (wie auf traditionellen Schulzeugnissen) handelt. Das Arbeitszeugnis ist eine (oft im Text ausformulierte) Arbeitsbestätigung und Referenz. 
sprachlich-kommunikative und didaktische Einheit" darstellt (Meier 2006: 193). Texte (sowohl geschriebene als auch gesprochene) sind insofern wichtig, da in ihnen Informationen, Sachverhalte, Meinungen und Argumente transportiert werden, die den sprachlich-situativen Rahmen einer fremden Sprache abbilden (vgl. Meier 2006: 193). Die bei der Arbeit mit und an Texten angewandten Praktiken des Lesens, Redens-Über-Texte und Schreibens sind mitsamt den dafür nötigen Fähigkeiten fest im Unterricht verankert (vgl. PortmannTselikas 2005: 3).

Wenn man davon ausgeht, dass mit dem Begriff Sprachkompetenz die menschliche Fähigkeit bezeichnet wird, die es dem Menschen ermöglicht, sich verbal auszudrücken und zu kommunizieren, dann kann man darauf schließen, dass die Textkompetenz die „Fähigkeit des produktiven und rezeptiven Umgangs mit schriftsprachlich geprägter Sprache" darstellt (SchmölzerEibinger 2011: 50).

Die Textkompetenz steht als Teilkompetenz der Sprachkompetenz der Interaktionskompetenz gegenüber, die den mündlichen Sprachgebrauch betrifft. (vgl. SchmölzerEibinger 2011: 49-50). Auch PortmannTselikas (2005: 2) setzt die Textkompetenz nicht mit der Sprachkompetenz gleich, da Erstere den erfolgreichen Umgang mit den Spezifika der geschriebenen Sprache, die sich in vielerlei Hinsicht von der gesprochenen Sprache abgrenzt, beschreibt. Es sind vor allem bildungssprachliche ${ }^{1}$ Elemente, durch die sich Texte in der Schule auszeichnen. Eine alltagssprachlich hohe Sprachkompetenz ist also auf keinen Fall mit einer hohen Textkompetenz im Bereich der Bildungssprache gleichzusetzen, da diese ganz andere Verstehensanforderungen stellt (vgl. Krumm 2007: 204).

Diesen (u. a. bildungssprachlichen) Spezifika der Schriftsprache entsprechend unterteilt SchmölzerEibinger (2011: 50) die Textkompetenz in folgende Teilkompetenzen, die untrennbar miteinander verbunden sind. Die ersten fünf Teilkompetenzen gelten als sprachenübergreifende und weitgehend transferierbare Fähigkeiten:

1. Kohärenzkompetenz: Sinnzusammenhänge in einem Text erkennen und selbst produzieren können;

2. Kontextualisierungskompetenz: Bedeutungen mit Hilfe des eigenen Kontextwissens in spezifische Kontexte einbetten können;

3. Kommunikationskompetenz: die kommunikative Funktion von Texten erkennen und Texte adressatengerecht produzieren können;

4. Textoptimierungskompetenz: Texte überarbeiten und verbessern können;

5. Strategische Kompetenz: Texte mit Hilfe von Lese- und Schreibstrategien erschließen und verarbeiten bzw. produzieren können;

6. Formulierungskompetenz: das, was man kommunizieren will, mit passenden sprachlichen Mitteln ausdrücken können;

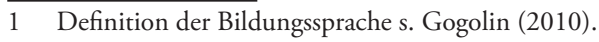


7. Textgestaltungskompetenz: einen sprachlich differenzierten und strukturierten Text produzieren können;

8. Textmusterkompetenz: konventionalisierte und kulturspezifisch geprägte Textmuster erkennen und produzieren können.

Zusätzlich nennt SchmölzerEibinger (2011: 50) noch die metatextuelle Kompetenz, welche die Fähigkeit benennt, über Lese-, Rezeptions- und Schreibprozesse einerseits und die Produkte (Texte) andererseits reflektieren, kommunizieren und diese bewerten zu können.

Ein wesentlicher Teil der Textkompetenz ist demnach das Textverstehen, welches nicht alleine durch den Zugriff auf die Informationen an der Textoberfläche erfolgt, sondern zusätzliches Zugreifen auf das bestehende (kulturspezifische) Wissen der Lernenden über Textsorten ${ }^{1}$ erfordert. Nur wenn die Lernenden in der Lage sind auch die Texttiefenstruktur zu erfassen und die globalen Sinnzusammenhänge eines Textes zu identifizieren, ist Textverstehen gewährleistet (vgl. SchmölzerEibinger 2011: 59-60). Die Produkte dieser Verstehensprozesse sind sog. „ReRepräsentationen“. Lernende filtern dabei (unter Anwendung von mentalen Modellen, die sie sich anhand von anderen Texten konstruiert haben) wichtige Informationen und Aspekte aus Texten heraus und verarbeiten diese wiederum zu neuen Texten, indem sie sie neu strukturieren und umformen. Dabei entsteht ein (kurzer) Text über einen Text.

Wenn die Lernenden hingegen nicht in der Lage sind von der Bedeutung der Textoberfläche zum tieferliegenden Textsinn zu gelangen, dann kommt es zu Verstehensproblemen und Verzerrungen der Re-Repräsentationen.

Aus diesen Überlegungen lässt sich ableiten, dass Lernende mit einer hohen Textkompetenz einen leichteren Zugang zu (Sach-) Texten und damit zu dem in ihnen vermittelten Wissen haben. Sie können Texte außerdem selbständig zum Lernen und zur Erarbeitung anderer Texte einsetzen. Lernenden mit niedriger Textkompetenz ist dieser Zugang oftmals verwehrt und sie können Texte nicht in ihren Lernprozessen nutzen (vgl. PortmannTselikas 2005: 1). Somit stellt die Textkompetenz in unserem Bildungssystem „eine der zentralen Ressourcen für Erfolg" (PortmannTselikas 2005: 1) dar und kann mitunter über Abschlüsse und Bildungskarrieren entscheiden.

\section{Förderung der Textkompetenz}

Wie oben angeführt, bedeutet die Textkompetenz eine Schlüsselkompetenz in der heutigen Gesellschaft. Gleichzeitig ist jedoch aus unterschiedlichen Erhebungen und Studien² bekannt, dass bei vielen Lernenden (selbst im Bereich Deutsch

1 Zur Abgrenzung und Definition von Text und Textsorte s. Káňa (2013: 107-119).

2 Zum Beispiel die PISA-Studie. 
als Muttersprache) diese wichtige Kompetenz nur mangelhaft entwickelt ist. Daraus resultiert ein großes Interesse an Fördermaßnahmen und -konzepten, die die Textkompetenz und ihre Teilbereiche (v. a. Lese- und Schreibkompetenz) herausbilden. Ihr Ziel ist, den Lernenden das notwendige Handwerkszeug zu vermitteln, mit dem sie ihre Lese-, Verstehens- und Schreibprozesse selbständig gestalten und optimieren können (vgl. Knapp 2012: 263). Das kann unter anderem dadurch geschehen, dass bei der Textarbeit Prozeduren und Strategien im Umgang mit Texten eingeübt werden, die generalisier- und übertragbar sind und somit zur Erschließung von anderen, schrittweise längeren und komplexeren Texten eingesetzt werden können. Zusätzlich soll ein Wissen über die Strukturen von „guten“ Texten erworben werden (vgl. PortmannTselikas 2005: 5-6).

Die Förderung der Textkompetenz sollte immer Hand in Hand mit der Förderung der bildungssprachlichen Fähigkeiten gehen. Ansätze und Vorschläge zur Sprach- und Textförderung müssen deshalb immer auch auf den Aspekt hin gestaltet und untersucht werden, ob und wie weit bildungssprachliche Fähigkeiten mitgefördert werden (dazu Krumm 2007: 200).

Ein Modell zur Förderung der Textkompetenz ist das 3-Phasen-Modell von SchmölzerEibinger (2011: 191), das als primäres Ziel die literale Förderung von Lernenden in mehrsprachigen Klassen verfolgt und sich auch gut für homogene Klassen der Fremdsprachenvermittlung eignet. Es soll die Lernenden dabei unterstützen, „die schriftsprachlichen Anforderungen im rezeptiven und produktiven Umgang mit Texten zu bewältigen und Texte verstehen, verarbeiten, verfassen und als ein Instrument des Lernens im Unterricht nutzen zu können“" (SchmölzerEibinger 2011: 191). Die von der Autorin vorgestellte literale Didaktik setzt dafür an der Basis von Verstehens- und Schreibprozessen an und zielt auf die Vermittlung und das Erlernen von basalen Strategien im Umgang mit Texten. Diese sollen auf mehr oder weniger komplexe Texte sowie unabhängig von ihren Inhalten und textsortenspezifischen Charakteristika angewendet werden können. Es wird Wert darauf gelegt, dass diese Vermittlung und Schulung in authentischen Sprachlernsituationen und nicht anhand von vereinfachten Texten geschieht (vgl. SchmölzerEibinger 2011: 176-178). Auf diese Weise können erfolgreich intensive Lese-, Verstehens- und Produktionsprozesse in Gang gesetzt werden, die über drei Phasen hinweg in immer anspruchsvoller werdenden Aufgaben vollzogen werden müssen. Zusätzlich zum Zusammenspiel von inhalts- und sprachbezogenen Aktivitäten und Aufgaben wird auch die metasprachliche und metakognitive Arbeit an Texten nicht vernachlässigt (vgl. SchmölzerEibinger 2011: 191-202).

Die drei Phasen des Modells (1. Wissensaktivierung, 2. Arbeit an Texten, 3. Texttransformation) sind genau aufeinander abgestimmt und bieten jeweils Aktivitäten und Aufgaben an, die die kooperative Bearbeitung (Partner- und Gruppenarbeit) von Texten ins Zentrum stellen. Im Folgenden wird das Modell von SchmölzerEibinger (2011: 191-193) verkürzt dargestellt. 
In der ersten Phase (1. Wissensaktivierung) sollen die Lernenden durch assoziatives Schreiben oder Sprechen ihr Vorwissen zu einem bestimmten Thema aktivieren. Als Sprech- oder Schreibanlass können Wörter, Bilder, ein Satz usw. dienen, anhand derer die Lernenden ihre Gedanken und Assoziationen spontan und frei aufschreiben bzw. darüber sprechen. So wird das Vorwissen der Lernenden für die späteren Aktivitäten und Arbeit am Text verfügbar gemacht.

In der nächsten Phase des Modells (2. Arbeit an Texten) sollen die Lernenden Texte aus verschiedenen Blickwinkeln und in verschiedene Kontexte eingebettet bearbeiten. Dies geschieht durch unterschiedliche Aktivitäten: Lesen, Schreiben, Interpretieren, Reflektieren, Diskutieren, Rekonstruieren und Überarbeiten. Diese Aktivitäten verteilen sich auch auf drei Phasen: (A) Textkonstruktion, (B) Textrekonstruktion, (C) Textfokussierung und Textexpansion. In der ersten Teilphase (A) sollen Textfragmente ergänzt und Lücken im Text geschlossen werden. Die Textrekonstruktion (B) bietet als Aktivität Lückentexte zu vervollständigen bzw. gelesene oder gehörte Texte möglichst genau wiederzugeben (Dictogloss). In den Aufgaben zur Textfokussierung (C) werden wichtige Inhalte eines Textes aufgegriffen und auf das Wesentliche reduziert bzw. wiedergegeben oder ein Text zusammengefasst.

Die dritte Phase (3. Texttransformation) setzt Texte in einen anderen Kontext ein, was eine Übernahme der Strukturen und Anpassung an ebendiese Kontexte erfordert. In dieser letzten Phase ist die Autonomie der Lernenden am stärksten gefordert, denn sie müssen ihre Lernprozesse tatsächlich selbst steuern und gestalten.

Dieses Modell stellt auch im Kapitel 5 die Grundlage für die Förderung der Textkompetenz in der hier gewählten Textsorte dar. Zuerst soll jedoch die Textsorte selbst, ihre Einbettung und ihre Eigenschaften vorgestellt werden.

\section{Textsorte Arbeitszeugnis}

\subsection{Rechtsgrundlagen}

Das Arbeitszeugnis ist eine durch Gesetzesvorgaben stark normierte und an Konventionen gebundene Textsorte. In Österreich ist die Erteilung des Arbeitszeugnisses in $\$ 1163$ ABGB und $\$ 39$ AngG, in Deutschland in $\$ 630$ BGB, $\$ 109$ GewO und $\$ 16$ BBiG und in Tschechien in Zákoník práce (2006): $\$ 313$ des Arbeitsgesetzes (Gesetz Nr. 262/2006 GB) ${ }^{1}$ geregelt.

Das österreichische ABGB (2017), $\$ 1163$ besagt, dass Arbeitnehmer/-innen bei Beendigung ihres Arbeitsverhältnisses (auf Verlangen) Anspruch auf ein schriftliches Zeugnis über die Dauer und Art der Dienstleistung haben, das gebührenfrei

1 Auf Spezifika und Unterschiede der Arbeitszeugnisse bzw. ihres tschechischen Pendants wird hier nicht weiter eingegangen. 
ausgestellt wird. Falls sich der/die Arbeitgeber/-in weigert, ein Arbeitszeugnis auszustellen, kann es der/die Arbeitnehmer/-in vor dem Arbeits- und Sozialgericht einklagen. AngG (2017) regelt den Zeugnisanspruch im $\$ 39$ mit identischem Wortlaut für Angestellte.

In der deutschen Gesetzgebung findet sich das Medium des Arbeitszeugnisses verankert (nicht so jedoch in Österreich): Das Zeugnis muss schriftlich verfasst sein. Die Erteilung in elektronischer Form wird laut Huber/Müller (2014: 17-18) gesetzlich ausgeschlossen. Zusätzlich zu der mehr (Deutschland) oder weniger (Österreich) gesetzlich geregelten Schriftformregelung ist festgelegt, dass das Arbeitszeugnis maschinenschriftlich oder mit dem Computer erstellt und auf Geschäftspapier (Firmenbogen) ausgestellt werden muss. Es darf keine Flecken, Durchstreichungen etc. enthalten und kann in einem solchen Fall vom Arbeitnehmer / von der Arbeitnehmerin zurückgewiesen werden, stellen Huber/Müller (2014: 23) fest.

Was den Inhalt betrifft, geben die Rechtsgrundlagen in Deutschland und Österreich nicht gleichermaßen Auskunft: Im österreichischen Gesetzbuch ist explizit vermerkt, dass „Eintragungen und Anmerkungen im Zeugnisse, durch die dem Dienstnehmer die Erlangung einer neuen Stellung erschwert wird, unzulässig [sind] “ (ABGB 2017). Im deutschen Recht ist festgelegt, dass das Zeugnis „klar und verständlich formuliert“ sein muss und dass es „keine Merkmale oder Formulierungen enthalten [darf], die den Zweck haben, eine andere als aus der äußeren Form oder aus dem Wortlaut ersichtliche Aussage über den Arbeitnehmer zu treffen" (GewO 2018).

Ebenfalls juristisch fixiert sind zwei weitere Prinzipien: das Gebot der „Wahrheit“ und des „Wohlwollens“. Ersteres, die Wahrheit der Beurteilung, ist ein wichtiger Grundsatz für die Erstellung des Arbeitszeugnisses nach einem Urteil des Bundesarbeitsgerichts von 1960 (vgl. Hesse/Schrader 2015: 10). Der/die Verfasser/in des Arbeitszeugnisses muss sich auf Tatsachen berufen und die vorgenommene Bewertung muss der Wahrheit entsprechen. Es dürfen keine Behauptungen oder Annahmen enthalten sein. Das zweite Gebot, das "Wohlwollen“, besagt (nach einem Urteil des Bundesgerichtshofs von 1963), dass der/die Verfasser/-in „wohlwollend“ formulieren muss, denn „das Formulierte darf dem/der Beurteilten das berufliche Fortkommen nicht ungerechtfertigt erschweren" (Hesse/Schrader 2015: 10-11).

Wie Huber/Müller (2014: 43) und Hesse/Schrader (2015: 11) bemerken, stehen diese beiden Gebote in einem gewissen Widerspruch zueinander und haben dazu geführt, dass sich eine eigene Zeugnissprache entwickelt hat, weshalb man heutzutage in Arbeitszeugnissen fast ausschließlich positive Formulierungen findet. Hierauf soll in Kapitel 4.5 näher eingegangen werden. 


\subsection{Adressaten/Adressatinnen, Thema und Funktion}

Arbeitszeugnisse sind eine Form der Personalbeurteilung und somit ist als primäre/r Adressat/-in der/die Arbeitnehmer/-in zu nennen, auf den/die das Arbeitszeugnis ausgestellt wird. Es stellt einen bleibenden Nachweis bzw. eine Bescheinigung für die Tätigkeit bzw. Anstellung bei einem/r bestimmten Arbeitgeber/-in innerhalb eines bestimmten Zeitraumes dar. Gleichzeitig ist das Arbeitszeugnis auch zentraler Bestandteil des Bewerbungsprozesses, da es ein Teil der Bewerbungsmappe ist und sich insofern auch an zukünftige Arbeitgeber/innen richtet. Diesen soll es dazu dienen, sich über die Persönlichkeit, Tätigkeiten, Qualitäten und das Verhalten des/ der potentiellen Mitarbeiters / Mitarbeiterin ein Bild zu machen und dessen/deren Potential zu beurteilen (dazu auch Huesmann 2008: 85).

Der/die Arbeitnehmer/-in und seine/ihre Leistungen bilden das Thema der Textsorte Arbeitszeugnis. Die Themenentfaltung ist deskriptiv, vorwiegend mit durchlaufendem Thema mit "Spezifizierung und Einordnung" (Brinker 2010: 56). Üblicherweise beginnt das Zeugnis mit Angaben zur Person und Arbeitsdauer. Weiter wird der/die Arbeitnehmer/in vorgestellt, seine/ihre Position, Tätigkeiten und Qualität ebendieser sowie die Arbeitsleistung und auch das Verhalten beschrieben. ${ }^{1}$

Im Unterschied zu anderen Textsorten rund um die Arbeitswelt sollten die Arbeitszeugnisse eine rein informierende Funktion (nach Brinker 2010: 98) erfüllen. Eine überzeugende Funktion wie z. B. in einer Bewerbung oder in einem Empfehlungsschreiben soll hier nicht vorkommen. Wie wir jedoch später zeigen, ist diese implizit oft in Arbeitszeugnissen beinhaltet.

\subsection{Arten von Arbeitszeugnissen}

Hesse/Schrader (2015: 8-14) nennen als wichtigste Typen von Arbeitszeugnissen die folgenden, welche nach inhaltlichen und zeitlichen Gesichtspunkten sowie der Art des Beschäftigungsverhältnisses eingeteilt werden:

- Einfaches Zeugnis,

- Qualifiziertes Zeugnis,

- Zwischenzeugnis,

- Berufsausbildungszeugnis,

- Praktikums-, Ferien-, Aushilfs- und Nebenjobzeugnis.

Das einfache Zeugnis wird in der Regel bei kürzeren Beschäftigungsverhältnissen bzw. weniger qualifizierten Tätigkeiten ausgestellt. Es bestätigt die Art und Dauer des Arbeitsverhältnisses und führt seine Beendigungsgründe und -modalitäten an.

1 Im Internet gibt es genügend Muster und Vorlagen mit dieser Struktur. Stellvertretend siehe beispielsweise https://www.arbeitszeugnis.io/arbeitszeugnis-vorlage/. 
Das qualifizierte Arbeitszeugnis, der gängigste Zeugnistyp, ist ausführlicher gestaltet und enthält zusätzlich zu den Informationen über Art und Dauer des Beschäftigungsverhältnisses eine genauere Angabe über die Tätigkeiten sowie eine Leistungs- und Verhaltensbeschreibung und -beurteilung des Arbeitnehmers / der Arbeitnehmerin. Es ist ein Standardbestandteil der Bewerbungsmappe, wird jedoch nur ausgestellt, wenn das Beschäftigungsverhältnis eine gewisse Zeit bestanden hat.

Das Zwischenzeugnis, dessen Anspruch gesetzlich nicht so eindeutig geregelt ist wie der des qualifizierten (End-) Zeugnisses, unterscheidet sich von diesem dahingehend, dass das Beschäftigungsverhältnis noch nicht beendet ist. Die letzten beiden Zeugnistypen unterscheiden sich durch die Art des Beschäftigungs- bzw. Ausbildungsverhältnisses und können laut Hesse/Schrader (2015: 13-14) einfach oder qualifiziert sein.

\subsection{Textaufbau und Textorganisation}

Wie in den letzten Absätzen bereits deutlich gemacht wurde, ist das Arbeitszeugnis eine formal und auch inhaltlich streng genormte und größtenteils auch gesetzlich geregelte Textsorte. Auch die Textorganisation (Titel, Anzahl und Anordnung der Teilthemen) ist laut BremerichVos (2000: 882) weitgehend festgelegt. Als Mindestumfang des Arbeitszeugnisses gilt eine Seite und als Optimalumfang eineinhalb bis zwei Seiten (Hesse/Schrader 2015: 20). Die einzelnen Bestandteile eines qualifizierten Arbeitszeugnisses können nach Hesse/Schrader (2015: 19-20) und Huber/Müller (2014: 54) wie folgt zusammengefasst werden:

- Überschrift: Zeugnis, Arbeitszeugnis, Zwischenzeugnis (nicht: Endzeugnis);

- Einleitung: Angaben zur Person (Name, Anschrift, Geburtsdatum und -ort des Arbeitnehmers / der Arbeitnehmerin), Beruf und Beschäftigungsdauer (Beginn und Beendigungsdatum);

- Unternehmensbeschreibung (fakultative Angabe);

- Tätigkeitsbeschreibung: Position, Hierarchieebene, Tätigkeitsmerkmale, Kompetenzen, Verantwortung sowie berufliche Entwicklung innerhalb des Unternehmens;

- Leistungsbeurteilung: Arbeitsbereitschaft, Arbeitsbefähigung, Arbeitsweise, Arbeitserfolg, Fachkönnen, Führungsleistung und andere mit der Ausübung des Berufs zusammenhängende Eigenschaften und Kompetenzen; abschließend zusammenfassende Leistungsbeurteilung;

- Beurteilung des persönlichen und sozialen Verhaltens: Verhalten gegenüber Vorgesetzten/Kollegen/Dritten, weitere persönliche und soziale Verhaltensaspekte; abschließend zusammenfassende Verhaltensbeurteilung; 
- Schlussformulierung, Schlussformel: Grund für die Ausstellung des Zeugnisses; weitere optionale Elemente: Gründe für die Beendigung des Arbeitsverhältnisses; Dankes-/Bedauernsformel, Zukunftswünsche;

- Administrative Angaben: Ausstellungsort und -datum, Unterschrift des/der Zeugnisausstellenden: Name des Zeugnisausstellenden zusätzlich maschinenschriftlich, Hinweis auf dessen/deren Position, Rechtsstellung. Das Ausstellungsdatum sollte womöglich identisch mit dem Austrittsdatum sein.

Diese einzelnen Textelemente können (je nach Arbeitszeugnis) mehr oder weniger ausführlich ausgeführt sein. Die angeführte Reihenfolge ist verbindlich. Hierfür sowie für das Arbeitszeugnis als Gesamtes gilt: Individuelle Abweichungen können negative Folgen haben, und zwar meist (nur) für den Beurteilten / die Beurteilte. Hesse/Schrader (2015: 20) betonen, dass eine Umstellung der Textabschnitte (z. B. Verhaltens- vor Leistungsbeurteilung) bereits als ein Hinweis auf eine Negativbewertung verstanden werden können. Die Schlussformel (Bedauerns-, Dank- und Wünsche-Formel) ist seit etwa 2010 nicht mehr obligatorisch. Die Gestaltung dieser letzten Passage ist seitdem (arbeitsgerichtlich gedeckt) dem/der Zeugnisaussteller/-in überlassen. Hesse/Schrader (2015: 18-19) fügen noch hinzu: „Die Begründung der Auflösung des Beschäftigungsverhältnisses war nie zwingend vorgeschrieben, findet sich aber in den meisten Arbeitszeugnissen“.

\subsection{Sprachliche Mittel und Codes}

Beim Arbeitszeugnis handelt es sich nicht um ein klassisches Zeugnis im Sinne eines Schulzeugnisses mit einer Benotung von 1-5 (bzw. 6). Die Bewertung entspricht zwar diesem Notenschlüssel, ist jedoch in Worten und in einer speziellen „Zeugnissprache" formuliert.

Arbeitszeugnisse werden in der Regel aus der „Wir-Perspektive“, also in der „Wir-Form“, geschrieben (Wir schätzen...; Wir danken...; Wir bestätigen...) und beziehen sich in der dritten Person Singular (Herr X, Frau Yoder Personalpronomen) auf die/den Beurteilte/-n.

Die verwendeten Tempusformen sind vom Typ des Arbeitszeugnisses abhängig. In vielen Arbeitszeugnissen werden jedoch Vergangenheitsformen (Präteritum und Perfekt) sowie Präsens gemischt verwendet. Eine verstärkte Verwendung des Präsens findet man in Zwischenzeugnissen, da in diesen Fällen das Arbeitsverhältnis noch nicht beendet ist. Unabhängig vom Zeugnistyp wird die Schlussformel immer im Präsens verfasst, da sich der/die Zeugnisaussteller/-in hier direkt an den/die Arbeitnehmer/-in richtet (Wir danken..., Wir hoffen...; Wir wünschen...; Wir bedauern...). Bei diesen Verben handelt es sich oft um expressive Verben, die die Sprachhandlungen des Dankens, Hoffens, Wünschens und BEDAUERNS ausdrücken. 
Arbeitszeugnisse sind vom Nominalstil geprägt und enthalten nicht selten auch übermäßig viel Fachwortschatz, der vor allem in der Tätigkeitsbeschreibung bzw. auflistung und auch in der Leistungsbeurteilung zu finden ist.

Typisch sind (vorrangig in der Leistungs- und Verhaltensbeurteilung) auch Quantifikatoren und Qualifikatoren, die durch attributive Konstruktionen realisiert werden (sehr, rasch, gewissenhaft, schnell, zielstrebig, umsichtig).

Bei der Durchsicht von Arbeitszeugnissen fällt recht schnell auf, dass eigentlich nur positive Formulierungen zu finden sind. Man könnte nun annehmen, dass die Zeugnisse durchgängig eine positive Beurteilung widerspiegeln. Dies ist jedoch nicht der Fall. Die Sprache von Arbeitszeugnissen kann und darf nicht mit dem alltäglichen Sprachgebrauch gleichgesetzt werden, denn ihr Verständnis ist schwer und nicht immer eindeutig. Arbeitszeugnisse können mitunter voll von „wohlwollenden“ Standardformulierungen sein, die etwas Anderes (Negatives) meinen, als sie auf den ersten Blick aussagen. Dies ist auf den Widerspruch zwischen Wahrheits- und Wohlwollens-Gebot zurückzuführen. Das Wohlwollens-Gebot (vgl. Kap. 4.1) hat dazu geführt, dass Negatives nicht explizit vermerkt werden darf, zahlreiche Standardformulierungen in Arbeitszeugnissen aber dennoch einer genauen Beurteilungsskala entsprechen. Wie Huesmann (2008: 77) schreibt, erlaubt uns diese Positiv-Skala-Technik, über eine Abstufung der positiven Bewertungen diese den Schulnoten zuzuordnen. Die wohl bekanntesten Standardformulierungen des Arbeitszeugnisses sind nach Hesse/Schrader (2015: 25) in der Tabelle 1 angeführt.

\begin{tabular}{|l|c|}
\hline $\begin{array}{l}\text { Er hat die ihm übertragenen Aufgaben stets zu unserer vollsten } \\
\text { Zufriedenheit ausgeführt }\end{array}$ & 1 \\
\hline$\ldots$ stets zu unserer vollen Zufriedenheit ausgeführt & $1-2$ \\
\hline$\ldots$ zu unserer vollsten Zufriedenheit & 2 \\
\hline$\ldots$ zu unserer vollen Zufriedenheit ausgeführt & 3 \\
\hline$\ldots$ zu unserer Zufriedenheit erledigt. & 4 \\
\hline$\ldots$ im Großen und Ganzen zu unserer Zufriedenheit erledigt. & $4-5$ \\
\hline $\begin{array}{l}\text { Er hat sich bemüht, die ihm übertragenen Aufgaben zu unserer } \\
\text { Zufriedenheit zu erledigen. }\end{array}$ & 5 \\
\hline
\end{tabular}

Tab. 1: Beurteilungsskala: Transformation vom Text zu Noten

Wie in der Gegenüberstellung der einzelnen Formulierungen ersichtlich wird, machen einzelne Wörter den Unterschied in der Benotung aus. Die häufigsten sprachlichen Mittel bei der Erzeugung von Nuancen in der Benotung sind Steigerungsformen und (Temporal) Adverbien: stets, immer, allzeit sind positiv; teilweise, im Wesentlichen, im Großen und Ganzen negativ. Auf diese Weise können in einem positiven Zeugnis durch feine Nuancen alle Schulnoten verwendet werden, 
ohne eine negative Formulierung zu benutzen. Janssen/Beden (2006: 45) bemerken, dass ein auf den ersten Blick positiv klingendes Zeugnis in Wahrheit ein recht differenziertes Leistungs- und Verhaltensbild des/der Beurteilten zeichnen kann.

$\mathrm{Zu}$ den Besonderheiten der Zeugnissprache gehören noch weitere Techniken mit Hilfe derer Kritik implizit formuliert werden kann. Einige sollen hier nach Jenssen/Beden (2006: 53-56) vorgestellt werden:

- Auslassungstechnik oder „beredtes Schweigen: Bestimmte Teilthemen gelten als obligatorisch und ihre Auslassung oder auch nur Abweichungen von den Standardformulierungen sind informativ zu verstehen.

- Reihenfolgetechnik: Werden Dritte (Kunden / Kundinnen) an erster Stelle und der Vorgesetzte an letzter erwähnt, kommt die Reihenfolgetechnik zur Anwendung und es wird auf einen „aufsässigen Mitarbeiter“ / eine „aufsässige Mitarbeiterin" verwiesen. Auch innerhalb der Tätigkeitsbeschreibungen gilt: Wenn weniger wichtige Tätigkeiten vor wichtigen genannt werden, dann werden letztere abgewertet.

- Passivierungstechnik: Die häufige Verwendung von passiver Formulierungen deutet auf eine "passive“ Mitarbeiterin / einen „passiven“ Mitarbeiter hin.

- Einschränkungstechnik: Einschränkende Zusätze und/oder Relativsätze (etwa ...erzielte meist gute Verkaufsergebnisse...) wirken stets negativ.

- Widerspruchstechnik: Bewusstes Konstruieren von Widersprüchen und Ungereimtheiten (...arbeitete nach Vorgaben selbständig...) versteht ein aufmerksamer Leser / eine aufmerksame Leserin sofort als eine vernichtende Kritik.

Dem/der Zeugnisausteller/-in stellt sich beim Verfassen eines Arbeitszeugnisses also grundsätzlich die Frage: Wie kann ich etwas tatsächlich Negatives wohlwollend beschreiben und dabei gleichzeitig dem Wahrheitsgebot entsprechen? Für den Rezipienten / die Rezipientin stellt sich hingegen die Frage: Meint der/die Zeugnisschreiber/-in, was er/sie sagt, und schreibt er/sie, was er/sie meint?

Aus kommunikationstheoretischer Sicht können die von den eben beschriebenen sprachlichen Mitteln und Techniken zusätzlich ausgedrückten Bedeutungen als Implikaturen ${ }^{1}$ bezeichnet werden. Für das Verstehen der expliziten und impliziten Bedeutungen eines Arbeitszeugnisses müssen also Informationen über die Kommunikationssituation, das gemeinsame Wissen, die Intentionen der Teilnehmer/-innen und die Funktion der Textsorte miteinbezogen werden.

1 Nach Grice das „tatsächlich Gemeinte, jedoch nicht Gesagte“ (mehr dazu in DUDEN-Grammatik 2016: 1176 und Fix/Poethe/Yos 2003: 21). 


\section{Das Arbeitszeugnis im Unterricht}

\subsection{Eignung für den DaF- und DaZ-Unterricht}

Auf den Stellenwert von Texten im Unterricht allgemein wurde schon in den Kapiteln 2 und 3 eingegangen. Hier soll nun der Frage nachgegangen werden, ob die Textsorte Arbeitszeugnis auch im Fremd- und Zweitsprachenunterricht eingesetzt werden könnte, und zwar zu dem speziellen Zweck der Textförderung. Wie wir im vorherigen Kapitel gezeigt haben, handelt es sich beim Arbeitszeugnis um eine auch gesetzlich stark genormte und abgegrenzte Textsorte, die nicht viel Handlungs- und Formulierungsspielraum bei ihrer Gestaltung und Rezeption lässt. Außerdem erfordert die spezielle Zeugnissprache (Elemente, die in Kapitel 4 genannt worden sind) eine recht hohe Sprach- und Textkompetenz. Man muss den Lernenden nicht unbedingt beibringen, wie man Arbeitszeugnisse entschlüsselt, da einfach auf die massenweise angebotene Ratgeberliteratur zurückgegriffen werden kann (siehe weiter). Darüber hinaus ist nicht zu erwarten, dass die Lernenden in absehbarer Zeit diese Textsorte selbst verfassen müssen. Es wäre somit einfach zu argumentieren, dass sich die Textsorte Arbeitszeugnis weniger für einen Einsatz im Fremdsprachenunterricht (DaF oder DaZ) eignet.

In den nächsten Absätzen soll aber gegenteilig argumentiert und der Einsatz von Arbeitszeugnissen im Unterricht und auch zur Förderung der Textkompetenz befürwortet werden. Im Kapitel zur Förderung wurde angesprochen, dass sich Texte unabhängig vom Schwierigkeitsgrad für die Arbeit im Unterricht und auch für die Förderung der Textkompetenz anbieten. Aus diesem Grund kann und soll auch Arbeitszeugnissen ein Platz im Unterricht und in der Förderung der Leseund Produktionskompetenz sowie des Textverstehens generell eingeräumt werden. Der Einsatz muss natürlich zielgruppengerecht (Erwachsene und Jugendliche) und nur auf einem hohen Sprachniveau erfolgen. In Profile Deutsch (Glaboniat et al. 2005) wird das Arbeitszengnis ab Niveau B1 rezeptiv und ab Niveau B2 produktiv angeführt.

Im Zusammenhang mit der Textkompetenz und ihren Fördermöglichkeiten ist oft die Rede von literarischen Texten und Sachtexten im schulischen Fachunterricht (Physik, Geographie, Geschichte etc.). Beim Arbeitszeugnis handelt es sich weder um das eine noch um das andere, sondern um einen Gebrauchstext. Es soll hier mit Meier (2006: 198) argumentiert werden, dass literarische Texte, Sach- und Fachtexte gleichermaßen zur „Förderung einer berufsvorbereitenden, allgemeinbildenden und interkulturellen Kompetenz" eingesetzt werden sollen. Wir sind der Ansicht, dass alle drei dieser spezifischen Kompetenzen mit Hilfe des Arbeitszeugnisses erfolgreich trainiert werden können. Auch Maiwald (2010: 55) hebt hervor, dass Schüler und Schülerinnen im Umgang mit Texten, die sie beim Übergang ins Erwachsenenleben unmittelbar betreffen, geschult werden müssen. Dies trifft auf das Arbeitszengnis 
ebenso zu wie auf die von ihm aufgezählten Textsorten Stellenanzeigen, Verträgen, Behörden- und Gesetzestexte.

Es bietet sich an, das Arbeitszeugnis gemeinsam mit dem Lebenslauf und dem Bewerbungsschreiben zu thematisieren. Alle diese Textsorten beziehen sich gleichermaßen auf die Arbeitswelt. Anhand des Arbeitszeugnisses kann jedoch (im Vergleich zu den beiden anderen Textsorten) die Lesekompetenz und das Textverstehen besser trainiert werden. Darüber hinaus kann mit dem Arbeitszeugnis auch auf kulturelle Unterschiede aufmerksam gemacht werden, da dieses in manchen Staaten in der in Deutschland und Österreich üblichen Form einfach nicht existiert, wie wir bereits angemerkt haben.

Zusammenfassend kann also gesagt werden, dass die Relevanz dieser Textsorte für das Arbeitsleben der Lernenden gegeben ist. Anhand des Arbeitszeugnisses können schrittweise die Konventionen der deutschsprachigen Arbeitswelt vermittelt werden. Die Lernenden machen sich auch mit einem kulturspezifischen und stark normierten Text vertraut, der sich zur Suche nach Pendants in der eigenen Sprachwelt sehr gut eignet.

An dieser Stelle soll hervorgehoben werden, dass die Arbeit anhand von sorgfältig ausgewählten Beispieltexten geschehen muss. Im besten Fall (falls die Lernenden dazu bereit sind) kann auch mit ihren eigenen Arbeitszeugnissen gearbeitet werden, vorausgesetzt, dies widerspricht nicht anderen Gesetzen (z. B. dem Datenschutzgesetz). Der Text soll je nach Zielgruppe (Alter, Sprachniveau, Motivation) kürzer oder länger sein und sollte einen Beruf bzw. Tätigkeiten beschreiben, die für die Lernenden relevant und interessant sind.

\subsection{Fokussierte Aspekte und sprachliche Mittel}

In Kapitel 4 wurde eine Übersicht über die wichtigsten strukturellen, sprachlichen und kommunikativen Charakteristika und Besonderheiten der Textsorte Arbeitszeugnis präsentiert. Diese Textsorte verlangt bereits beim Einstieg eine hohe Textkompetenz und ein gutes Textmusterwissen, deswegen eignet sich die Arbeit damit erst ab der Niveaustufe B2. Um Arbeitszeugnisse umfassend richtig verstehen und deuten zu können, müssen selbst Muttersprachler und Muttersprachlerinnen in der Tat auf die Ratgeberliteratur zurückgreifen.

Das Ziel des Einsatzes im Unterricht soll jedoch nicht die vollständige und richtige Interpretation eines Arbeitszeugnisses sein, sondern vielmehr die Vermittlung von Strategien zur Förderung der Textkompetenz und ihrer Teilkompetenzen, wie im Kapitel 3 angeführt wurde.

Auf jeden Fall sollten die Typologie der Zeugnisse und die besondere Funktion des Arbeitszeugnisses thematisiert werden. Lernende können dazu einen persönlichen Bezug herstellen, denn sie sind laufend mit Benotung konfrontiert. 
Anhand des Arbeitszeugnisses lernen sie somit ein anderes System der Benotung und Beurteilung kennen, in dem Negatives nicht explizit verschriftlicht wird.

Des Weiteren soll unbedingt der pragmatische Aspekt hervorgehoben werden: Die Diskrepanz zwischen der Intention des Verfassers / der Verfasserin (KRITIK) und der sprachlichen Oberfläche (Lов) kann hier extrem groß sein. Dieser Aspekt sollte auch mit Normen und pragmatischen Aspekten in anderen Ländern verglichen werden.

Da die Gesetzestexte, die das Arbeitszeugnis regeln, recht einfach zu verstehen sind, können auch sie im Unterricht eingesetzt und thematisiert werden. Auch hier bietet sich ein Vergleich mit den Gesetzen anderer Länder an. Auf die beiden Prinzipien der „Wahrheit" und des „Wohlwollens“ muss auf jeden Fall hingewiesen werden.

Ebenfalls sollen die Sprachhandlungen Informieren und Beurteilen anhand der Frage „WAS macht der Text?" thematisiert und diskutiert werden. Damit geht die Frage nach dem „WIE informiert und v. a. WIE beurteilt der Text?“ einher. Die NichtVerwendung von negativen Formulierungen und Bewertungen kann in einzelnen Aktivitäten gut herausgearbeitet werden. Den Lernenden kann auf diese Weise vermittelt werden, dass bei der Rezeption von Arbeitszeugnissen einfaches Textverstehen nicht ausreicht, sondern dass der/die Rezipient/-in interpretieren muss, was sich hinter der wörtlichen Bedeutung der Ausdrücke und Phrasen verbirgt (verbergen kann). Dabei hilft auch die Positiv-Notenskala (s. Tab. 1). Dazu gibt es viele Beispiele und Materialien (Hesse/Schrader 2010, einschlägige Internetseiten). Somit können die verschiedenen Les und Interpretationsarten in einem spielerischen Kontext trainiert und geübt werden, immer mit der grundsätzlichen Frage im Hintergrund: „Meint der Zeugnisaussteller / die Zeugnisausstellerin, was er/sie schreibt, und schreibt er/sie, was er/sie meint?" Den Lernenden soll deutlich gemacht werden, dass ein einzelnes Wort über die (Gesamt-) Bedeutung entscheiden kann und somit genau gelesen werden muss.

Auch für die Wortschatzarbeit ist das Arbeitszeugnis aufgrund seines oft verwendeten Fachwortschatzes geeignet.

Weniger eignen sich jedoch die einzelnen Techniken (z. B. Auslassungstechnik, Reihenfolgetechnik), da sie sehr schwer zu identifizieren sind. Wenn, dann ist ihre Funktionsweise nur anhand einer vorgefundenen Formulierung zu thematisieren oder für Textarbeit auf hohen Niveau- und Kompetenzstufen angebracht.

\subsection{Textsortenadäquate Fördermöglichkeiten}

Bei der Erstellung der nachfolgenden Fördermöglichkeiten und -aufgaben wird nach dem 3PhasenModell von SchmölzerEibinger (2011) vorgegangen. Manche der vorgestellten prototypischen Aufgaben (s. SchmölzerEibinger 2011:203) eignen sich aufgrund der Besonderheiten der Textsorte Arbeitszeugnis weniger für den Einsatz, 
andere können jedoch problemlos übernommen und für diese Textsorte adaptiert werden. Das Modell wurde ausgewählt, da es - wie in Kapitel 3 genauer beschrieben wurde - aufgrund seines besonders umfassenden Anspruches der Förderung der Textkompetenz alle Teilkompetenzen berücksichtigt. Die unten vorgestellten Fördermöglichkeiten müssen nicht unbedingt im schulischen Kontext angesiedelt sein, sondern sie können auch in außerschulischen Unterrichtssituationen (DaZKurse, Berufsvorbereitungskurse etc.) eingesetzt werden. Wichtig ist immer die spezifische Zielgruppe, an die die einzelnen Aktivitäten und Aufgaben angepasst werden müssen.

Bei der Suche nach Beispieltexten kann auf die Ratgeberliteratur (in Buchform und auch im Internet) zurückgegriffen werden. Vor allem Hesse/Schrader (2015 und 2010) bieten sich für die Auswahl an, da sie schlecht beurteilende Arbeitszeugnisse kommentieren und einer besser urteilenden Version gegenüberstellen.

Eine sehr ausführliche Auflistung von Textbausteinen (mit Einteilung in Notenskala) für alle Textabschnitte findet sich bei Knobbe/Leis/Umnuß (2014). Somit lässt sich die Arbeit mit Arbeitszeugnissen im Unterricht an unterschiedliche Niveaus und Schwierigkeitsgrade anpassen. In den nachfolgenden Aufgaben wird an keiner Stelle von den Lernenden verlangt, selbst ein vollständiges, normgerechtes Arbeitszeugnis zu verfassen.

\subsubsection{Vorschläge für die Fördermöglichkeiten}

Wie bereits angekündigt, stützen sich unsere Vorschläge auf das 3-Phasen-Modellvon SchmölzerEibinger (2011) mit dem Ziel, eine Basis für eine zukünftige empirische Untersuchung zu schaffen, in der die Funktionsfähigkeit dieses Modells anhand der Beispieltextsorte Arbeitszengnis überprüft werden kann.

\subsubsection{Phase 1: Wissensaktivierung}

In dieser Phase kann recht problemlos auf die Aufgabentypologie von SchmölzerEibinger (2011: 204) zurückgegriffen werden, denn das assoziative Schreiben und das assoziative Sprechen können gleichermaßen für das Arbeitszeugnis eingesetzt werden.

Das Ziel dieser Phase ist die Aktivierung des Vorwissens der Lernenden und die Abrufung von vorhandenen Schreibkenntnissen und deren „In-Bezug-Setzen“ zu den neu erworbenen Wissen (vgl. SchmölzerEibinger 2011: 192). Der Fokus in dieser Phase soll auf der Textsorte und weniger auf den inhaltlichen Aspekten liegen. Es soll also nicht in erster Linie der Wortschatz (Aufgaben, Tätigkeiten) einzelner Berufe thematisiert werden, sondern die Textsorte Zeugnis allgemein und ihre pragmatischen Komponenten: Sprechsituation, Adressaten / Adressatinnen, Intention und Funktion. In manchen Kursen muss das Spezifikum dieser 
Textsorte näher erklärt werden. ${ }^{1}$ Nur bei Lernenden, die bereits mit der Textsorte Arbeitszeugnis vertraut sind, kann von Beginn an das Arbeitszeugnis thematisiert und auch verlangt werden, dass sie ihre Erfahrungen, ihre Gedanken und ihr Wissen zu dieser spezifischen Unterkategorie von Zeugnissen beschreiben. Dies kann durch assoziatives Schreiben geschehen: Anhand eines Wortes (Zeugnis bzw. Arbeitszeugnis), eines Bildes (Situation, in der ein Zeugnis erteilt wird, Bild eines Zeugnisses) oder eines Gegenstandes (tatsächliches Zeugnis) soll die Textsorte Zeugnis thematisiert werden. Die Lernenden sollen nun frei darauf losschreiben und alles, was ihnen zum Thema Zeugnisse (Erfahrungen, Gefühle, Situationen etc.) einfällt, aufschreiben. Als Zeitrahmen für diese Aktivität sind je nach Zielgruppe zwischen 5-10 Minuten zu wählen. Das Schreiben geschieht in Einzelarbeit, anschließend werden die Texte einander vorgelesen (Partnerarbeit) und ein gemeinsamer Text wird verfasst. Diese Texte können dann zum Gegenstand eines Text-Wettbewerbes werden, vor dem sich die gesamte Gruppe/Klasse auf eine bestimmte Anzahl an Textkriterien einigt, anhand derer die einzelnen Texte beurteilt werden. In einer abschließenden Gruppenarbeit können außerdem die Erfahrungen in den vorangegangenen Schreibprozessen thematisiert und besprochen werden.

Alternativ können beim assoziativen Sprechen ein Wort, ein Bild oder mehrere Bilder (verschiedene Zeugnisse, aber besser verschiedene Situationen, in denen Zeugnisse vorkommen) eingesetzt werden. In Partnerarbeit wird in letzter Variante ein Bild ausgewählt und etwa fünf Minuten (gruppen- und niveauabhängig) darüber vor der restlichen Gruppe gesprochen, ohne dass der Sprecher/die Sprecherin dabei vom Kursleiter/von der Kursleiterin unterbrochen wird. Danach stellen die Kollegen/Kolleginnen drei Aspekte heraus, die ihnen besonders gefallen haben oder die sie bei dem Gehörten als besonders wichtig bewerten. Anschließend soll dazu in Gruppenarbeit ein Text verfasst werden, in dem die ausgesuchten Aspekte mit eigenen Ideen und Gedanken (das Bild und die Aspekte betreffend) verbunden werden. Optional kann eine Person aus der Gruppe den Schreibprozess beobachten und später ihre Beobachtungen und Erkenntnisse mitteilen. Anschließend werden die Texte innerhalb der Gruppen ausgetauscht und beurteilt. Die Texte können anhand der Rückmeldungen überarbeitet werden.

Ziele dieser Aktivitäten sind das freie Schreiben und Sprechen, das Einbringen eigener Erfahrungen, Gedanken und Vorstellungen. Im Zentrum stehen die Funktion und der Zweck von Zeugnissen. Vorschläge für konkrete Fragestellungen: Was ist/macht ein Zeugnis? Wann/In welchen Situationen bekommt man ein Zeugnis? Welche Erfahrungen habt ihr mit Zeugnissen gemacht? Warum sind euch gute Noten wichtig? Auf diese Weise werden die Lernenden auf den Kommunikationskontext

1 Unter Umständen wäre sinnvoll die Textsorten Arbeitszeugnis und Empfehlungsschreiben zu vergleichen und voneinander abzugrenzen. 
und die verschiedenen möglichen situativen Einbettungen der Textsorte aufmerksam gemacht und können auch ihre persönlichen Erfahrungen und Gefühle einbringen.

\subsubsection{Phase 2: Arbeit an Texten}

\section{A) Textkonstruktion}

In dieser Phase geht es darum, Texte zu erweitern oder zu vervollständigen. Die Lernenden müssen anhand ihres eigenen Wissens Lücken schließen (vgl. SchmölzerEibinger 2011: 195). Es werden Textfragmente vorgegeben, die als inhaltliche und auch sprachliche Vorgaben dienen.

Zum Beispiel kann der Textanfang eines Arbeitszeugnisses (Überschrift, Einleitung) vorgegeben werden, an dem die Lernenden dann weiterschreiben müssen. Die Einleitung enthält Informationen zur Person und gibt den Lernenden zu erkennen, dass sie die Arbeit dieser Person zu bewerten haben. Lernende, die nicht mit der Textsorte Arbeitszeugnis vertraut sind, werden dies vielleicht den klassischen Schulzeugnissen entsprechend tabellarisch und mit Schulnoten tun. Diejenigen, die mit der Textsorte vertraut sind und den gehobenen Stil kennen, können sich darin erproben, diesen Stil selbst zu verwenden. In dieser Phase geht es nicht darum, der „Norm“ entsprechend zu schreiben, sondern der Kreativität und Phantasie freien Lauf zu lassen.

Alternativ kann das Textende eines Arbeitszeugnisses vorgegeben werden (Unterschrift, Schlussformel und Verhaltensbeurteilung). Die Lernenden müssen dann anhand dieses Abschnitts den Textanfang formulieren.

Auch ein Vorgeben des Textanfangs und -endes ist möglich (Einleitung und Schussformel, evtl. weitere Zeugnisabschnitte).

Wie schon erwähnt ist das Ziel dieser Aktivitäten nicht, dass die Lernenden den Textsortennormen gemäß agieren und schreiben. Sie sollen stattdessen mit dem Stil und dem Aufbau des Arbeitszeugnisses vertraut gemacht werden, denen sie sich anpassen sollen.

Nach diesen Aktivitäten in Partnerarbeit sollen dann in Gruppenarbeit die Texte verglichen werden und gemeinsam ein neuer Text erstellt oder auch die eigenen Texte überarbeitet werden. Am Ende erhalten die Lernenden den Originaltext und können ihre Arbeitszeugnisse mit dem Original-Arbeitszeugnis vergleichen.

\section{B) Textrekonstruktion}

In dieser Phase geht es darum, Texte, die die Lernenden gehört oder gelesen haben, möglichst genau zu rekonstruieren (vgl. SchmölzerEibinger 2011: 197). Dabei soll der Schwierigkeitsgrad so gewählt werden, dass einzelne Formulierungen und Wörter nicht leicht behalten werden können. Hierfür eignet sich das Arbeitszeugnis 
mit seinen komplizierten Formulierungen recht gut. Es sollte jedoch ein anderer Beispieltext als in der vorangegangenen Phase verwendet werden, also ein Text, der den Lernenden noch unbekannt ist. Diesen (oder auch nur einen bestimmten Zeugnisabschnitt) kann man in Form eines Dictogloss den Lernenden vorlesen. Diese müssen dann in Gruppenarbeit den Text möglichst genau wiedergeben und dabei die Lücken anhand ihres eigenen Wissens schließen. Optional kann auch hier wieder eine Person den Schreibprozess beobachten und anschließend kommentieren. Folgend können die Texte miteinander verglichen und Verbesserungsvorschläge gemacht werden.

Geeigneter als ein Dictogloss scheint für das Arbeitszeugnis jedoch das Textpuzzle: Die Lernenden erhalten die einzelnen Absätze eines Zeugnisses ausgeteilt, sie müssen sie lesen und den anderen mitteilen, worum es in ihrem Absatz geht, z. B. "Hier wird eine Person vorgestellt." "Hier bedankt sich der Chef beim Arbeiter" „Hier wird beschrieben, was die Frau alles gearbeitet hat". Dann sollen die Absätze in der Gesamtgruppe in eine sinnvolle Reihenfolge gebracht werden.

Die Lernenden haben an dieser Stelle wohlgemerkt erst ein Arbeitszeugnis in vollem Umfang gesehen (im Rahmen der Unterrichtseinheit); die einzelnen Abschnitte und der Aufbau von Arbeitszeugnissen wurden noch nicht genauer thematisiert. Es handelt sich bei dieser Übung also um keine Überprüfung des Lernerfolgs, sondern um eine Aktivierungsübung, bei der die Lernenden anhand des in den vorangegangenen Aktivitäten gesammelten Wissens und ihrer vorläufigen Eindrücke von der Textsorte ihren Vermutungen zum Textaufbau und der Anordnung der Teilthemen anstellen. Es kann auch verlangt werden, den einzelnen Abschnitten Überschriften zu geben.

Am Ende soll ein zusammenhängender und sinnvoller Text entstehen, der mit dem Original-Arbeitszeugnis verglichen werden kann. An dieser Stelle ist es leicht möglich, dass die Lernenden einzelne Teilthemen (Leistungs- und Verhaltensbeurteilung) anders anordnen, als dies in der Vorlage der Fall ist. Dies stellt kein großes Problem in Hinblick auf die Kohärenz, sondern nur in Hinblick auf die Regelungen dar, die die Reihenfolge fest vorgeben. Darauf muss spätestens nach dieser Aktivität hingewiesen werden.

\section{C) Textfokussierung und Textexpansion}

Anschließend wird mit einem vollständigen Original-Arbeitszeugnis gearbeitet. Das Ziel dieser Teilphase ist es, die Aufmerksamkeit der Lernenden auf den Informationsgehalt des Textes und die besondere Struktur von Arbeitszeugnissen zu lenken sowie das Gewichten und die Wiedergabe von Informationen zu trainieren. Hierfür müssen inhaltliche Aspekte der Textsorte Arbeitszengnis ebenso behandelt werden wie die spezifischen Normen, die für diese Textsorte gelten. 
In dieser Phase kann von den Lernenden bereits verlangt werden, ein Arbeitszeugnis (in einem oder mehreren Sätzen) zusammenzufassen. Hierfür müssen die Lernenden die einzelnen Teilthemen des Textes bestimmen und nach ihrer Relevanz ordnen. Dabei zählt auch der Gesamteindruck, den die Lernenden vom Text gewonnen haben. Die Zusammenfassungen werden dann innerhalb der Gruppe verglichen und es können Verbesserungsvorschläge gemacht werden.

Die nachfolgenden Aktivitäten konzentrieren sich auf die sprachlichen Eigenschaften und Mittel der Textsorte Arbeitszeugnis. Eine Aktivität kann z. B. darin bestehen, die Schlüsselwörter in einem Arbeitszeugnis zu finden und anhand dieser Schlüsselwörter einen Text zu schreiben. Eine weitere Aufgabe könnte darin bestehen, die Kernaussagen und Kerninhalte des Textes zu finden oder diejenige Passage in einem Arbeitszeugnis zu bestimmen, die am besten ausdrückt, worum es in dem Zeugnis geht. Dies kann anschließend in der Gruppe diskutiert werden.

Auch könnte eine Aktivität darin bestehen, schwierige Textpassagen zu finden, die von den Lernenden nicht gänzlich verstanden werden. Eine Auswahl solcher Passagen könnte dann in der Gruppe verglichen werden, wobei sich die Lernenden gegenseitig erklären, warum sie die jeweiligen Passagen ausgewählt haben. Anschließend könnten die Textpassagen mit eigenen Worten umformuliert werden.

Als eine weitere, besonders wichtige Aufgabe könnten die Lernenden den Auftrag erhalten, alle Formulierungen, die eine positive (grün) und negative (rot) Wertung ausdrücken, zu markieren und in eine Tabelle einzutragen. Es soll dann diskutiert werden, was den Lernenden an diesen Ausdrücken auffällt. Danach kann eine Textpassage mit vielen positiven Formulierungen ausgewählt werden, die die Lernenden ins komplette Gegenteil umsetzen sollen, wobei ausschließlich negative Formulierungen verwendet werden dürfen.

In dieser Phase bietet sich ganz besonders die kontrastive Arbeit mit zwei Arbeitszeugnissen an - mit einem schlecht urteilenden und mit einem gut urteilenden (bzw. mit einer verbesserten Version eines schlecht urteilenden Zeugnisses). Da das Verbessern eines Arbeitszeugnisses ein sehr komplexes Unterfangen ist, sollten damit nicht die Lernenden beauftragt werden. Sie könnten aber anhand von zwei sehr ähnlichen Texten die feinen Unterschiede in den Formulierungen herausarbeiten und diese einander gegenüberstellen. Dies kann nach inhaltlichen Gesichtspunkten (Tätigkeiten, Verhalten etc.) oder abschnittsweise geschehen.

Die oben beschriebenen Aktivitäten trainieren zusätzlich den Wortschatz der Lernenden. Anschließend kann über den Ablauf der Vorgänge diskutiert oder ein Text geschrieben werden, in dem erklärt und begründet wird, welches das Arbeitszeugnis mit der besseren Beurteilung ist.

Es können auch einzelne Standardformulierungen (in allen Nuancen) ausgeteilt werden, die die Lernenden dann einer Notenskala zuordnen müssen. Auf diese 
Weise kann bewusst gemacht werden, wie vermeintlich positive Formulierungen sich recht rasch in weniger positive verwandeln können.

Als weitere Aktivität bietet sich an, die Lernenden eine Checkliste mit den basalen Bausteinen eines Arbeitszeugnisses erstellen zu lassen und ihnen dann ein Arbeitszeugnis zu präsentieren, auf das sie die Liste anwenden sollen. $\mathrm{Zu}$ diesem Zweck können bestimmte Abschnitte aus Arbeitszeugnissen gelöscht werden. Die Lernenden nehmen somit die Rolle von Experten/Expertinnen ein, die Arbeitszeugnisse auf ihren Aufbau und Umfang hin bewerten. Es sollen die Stellen vermerkt werden, an denen Wichtiges fehlt, und diese dann von den Lernenden ergänzt werden. Danach soll das ergänzte Zeugnis mit dem Originaltext verglichen werden.

Die Aufgaben zur Textexpansion beschreiben also Aktivitäten, in denen die bereits zusammengestellten Texte (bzw. Elemente von ihnen wie Textteile und Sätze) weiter ausgebaut werden und beispielsweise in einen Zeitungsartikel, einen Wikipedia-Artikel oder einen Brief eingebaut bzw. verwandelt werden.

\subsubsection{Phase 3: Texttransformation}

In der letzten Phase „[...] sind die Lernenden gefordert Bedeutungen aus dem Kontext herauszulösen, in einen anderen Kontext zu transformieren und neu aufzubauen " (SchmölzerEibinger 2011: 200). Als Aktivität bietet sich hier an, selbst ein Arbeitszeugnis zu verfassen, das sich aber nicht auf eine tatsächliche berufliche Tätigkeit bezieht, sondern zum Beispiel auf die Arbeit im Unterricht (Mitarbeit, Tätigkeiten, Aktivitäten, Leistung, Verhalten gegenüber Kollegen/Kolleginnen, Lehrpersonen usw.).

Des Weiteren können die Lernenden ein Arbeitszeugnis in eine Beschreibung vom Tagesablauf der beurteilten Person umformulieren, einen Wikipedia-Artikel zum Thema Arbeitszeugnis erstellen oder einen Brief an eine/n Freund/-in verfassen, in dem sie ihm/ihr erklären, warum er/sie das Arbeitszeugnis besonders genau analysieren soll.

Die produzierten Texte können zum Schluss miteinander verglichen und kommentiert werden. Die Überarbeitung der Texte kann wieder von einem/r Lernenden beobachtet und danach kommentiert werden.

\subsubsection{Zusatzaktivitäten}

Bei der Arbeit mit Arbeitszeugnissen eignet sich auch integrative Textarbeit mit anderen Textsorten, zum Beispiel einem Zeitungsartikel über die Arbeitswelt oder über eine Person, die vor dem Arbeitsgericht ihr Arbeitszeugnis einklagt. 
Ebenso bieten sich Rechercheaufgaben an, in denen die Lernenden den Auftrag erhalten, Bücher und Internetseiten zu suchen, in denen Tipps zum Verstehen und Entschlüsseln von Arbeitszeugnissen gegeben werden.

\section{Fazit}

Auch wenn man auf den ersten Blick meinen könnte, dass sich das Arbeitszeugnis aufgrund seiner Komplexität und seines streng normierten Charakters weniger für den Einsatz im DaF/DaZUnterricht eignet, so lässt sich doch festhalten, dass auch diese Textsorte aus mehreren Gründen relevant ist.

Erstens handelt es sich um eine Textsorte, die spezifisch für die deutschsprachige Welt ist.

Zweitens eignet sich auch der Sachtext Arbeitszeugnis gut für die Arbeit im Unterricht und kann dazu genutzt werden, die Textkompetenz und deren einzelne Teilkompetenzen auszubauen und zu trainieren. Das Arbeitszeugnis kann ab Niveaustufe B1 grundsätzlich immer dann thematisiert und eingesetzt werden, wenn die Arbeitswelt oder Themen wie Bewerbung und Bewerbungsverfahren und die Textsorten Lebenslauf und Bewerbungsschreiben behandelt werden.

Wie in Kapitel 4 deutlich wurde, zeichnet sich die Textsorte Arbeitszengnis durch zahlreiche spezifische Merkmale aus, was bedeutet, dass die Arbeit mit Arbeitszeugnissen im Unterricht wohlüberlegt und gut vorbereitet erfolgen muss. Sie ist aber gerechtfertigt, da das Arbeitszeugnis ein relevantes Dokument für das Leben einer jeden Person ist, die arbeitet oder sich um eine Stelle bewirbt. Die Bedeutung der Textsorte sollte den Lernenden auf jeden Fall vermittelt und der Umgang mit ihr (Lesen - Verstehen - Bewerten - Produzieren) anhand verschiedener und abwechslungsreicher Aufgaben und Aktivitäten geübt und trainiert werden.

Das 3-Phasen-Modell von SchmölzerEibinger hat sich, wie in Kapitel 5 gezeigt wurde, dahingehend und auf die Erstellung von Aufgaben zur Förderung der Textkompetenz bezogen als sehr geeignet erwiesen. Die prototypischen Aktivitäten konnten bis auf kleine Ausnahmen problemlos auf die Textsorte Arbeitszengnis übertragen werden und es wurde über die einzelnen Phasen hinweg deutlich, dass das Modell (zumindest theoretisch) tatsächlich auf eine umfassende Förderung aller Teilkompetenzen der Textkompetenz abzielt.

Es können natürlich nicht alle sprachlichen Besonderheiten der Textsorte oder Strategien, die für das Verstehen von Arbeitszeugnissen nötig sind, im Unterricht (ausreichend) thematisiert werden. Das primäre Ziel des Einsatzes von Arbeitszeugnissen im Unterricht soll, wie mehrmals hervorgehoben wurde, ja auch nicht das Verstehen ganzer Arbeitszeugnisse und das Entschlüsseln verschiedener Formulierungsvarianten sein. Mit diesem Artikel wollten wir lediglich zeigen, wie sehr sich auch diese besondere Textsorte für die Entwicklung von Strategien zur Arbeit mit und an Texten eignet. 


\section{Literaturverzeichnis}

ABGB (2017): Allgemeines bürgerliches Gesetzesbuch, $\$ 1163$. In Kraft seit 1917, Berücksichtigter Stand der Gesetzgebung: 15.12.2017. https://www.jusline.at/gesetz/ abgb/paragraf/1163 (15.03.2018).

AngG (2017): Angestelltengesetz, $\$$ 39. In Kraft seit 1921, Berücksichtigter Stand der Gesetzgebung: 16.11.2017. https://www.jusline.at/gesetz/angg/paragraf/artikel1zu39 (15.03.2018).

BremerichVos, Albert (2000): Der Einfluss der Textlinguistik auf die Ratgeberliteratur. In: Brinker, Klaus u.a. (Hrsg.): Text-und Gesprächslinguistik. 1. Halbband. Berlin/ New York: de Gruyter. S. 877- 884.

Brinker, Klaus (2010): Linguistische Textanalyse. Eine Einführung in Grundbegriffe und Methoden. Berlin: Erich Schmidt.

Dejure.org. $\$ 109$ GewO. In: https://dejure.org/gesetze/GewO/109.html (17.09.2017).

Dudenredaktion (o. J.): Duden online. http://www.duden.de (21.03.2018).

Fix, Ulla / Poethe, Hannelore / Yos, Gabriele (2003): Textlinguistik und Stilistik für Einsteiger. Ein Lehr-und Arbeitsbuch. Frankfurt a. M. et. al.: Peter Lang.

GewO (2018): Gewerbeordnung, $\mathbb{S} 109$ Zeugnis. Vorschrift eingefügt durch das Dritte Gesetz zur Änderung der Gewerbeordnung und sonstiger gewerberechtlicher Vorschriften vom 24.08.2002 (BGBl. I S. 3412), in Kraft getreten am 01.01.2003. https://dejure.org/ gesetze/GewO/109.html (15.03.2018).

Glaboniat, Manuela / Müller, Martin / Rusch Paul / Schmitz, Helen / Wertenschlag, Lukas (2005): Profile Deutsch. Gemeinsamer europäischer Referenzrahmen; Lernzielbestimmungen, Kannbeschreibungen, kommunikative Mittel; Niveau A1 - A2, B1 - B2, C1 - C2. CD-ROM Version 2.0 mit Begleitbuch. Berlin / München: Langenscheidt.

Gogolin, Ingrid (2010): Bildungssprache. In: Barkowski, Hans / Krumm, Hans-Jürgen (Hrsg.): Fachlexikon Deutsch als Fremd- und Zweitsprache. Tübingen / Basel: Francke. S. 29.

Hesse, Jürgen / Schrader, Hans Christian (2010): Training Arbeitszeugnis. Schreiben Interpretieren Verhandeln. Frankfurt a. M.: Eichborn.

Hesse, Jürgen / Schrader, Hans Christian (2015): Das perfekte Arbeitszeugnis. Standards, Formulierungen, Beispiele. Hallbergmoos: Stark.

Huber, Günter / Müller, Waltraud (2014): Das Arbeitszeugnis in Recht und Praxis. Die rechtlichen Grundlagen, Formulierungshilfen mit Textbausteinen, Musterzeugnisse, Zeugnisanalysen. Freiburg / München: Haufe.

Huesmann, Monika (2008): Arbeitszeugnisse aus personalpolitischer Perspektive. Gestaltung, Einsatz und Wahrnehmungen. Wiesbaden: Gabler Edition Wissenschaft.

Janssen, Verena / Beden, Manfred (2006) Arbeitszeugnisse. Richtig deuten - perfekt formulieren. GU Ratgeber. München: Gräfe und Unzer.

Jusline.at. \$1163 ABGB. In: https://www.jusline.at/gesetz/abgb/paragraf/1163 (17.09.2017). Káňa, Tomáš (2013): Texte, Textsorten und Texttypen. In: Sorger, Brigitte / Káňa, Tomáś / Janíková, ,Vèra / Reitbrecht, Sandra / Brychová, Alice (Hrsg.): Schreiben in mehreren Sprachen: Deutsch nach Englisch: Mehrsprachigkeit und ihr Einfluss auf die Textkompetenz. Brno: Tribun EU.

Knapp, Werner (2012): Förderunterricht in der Sekundarstufe. Welche Lese- und Schreibkompetenzen sind nötig und wie kann man sie vermitteln? In: Ahrenholz, Bernt (Hrsg.): Deutsch als Zweitsprache. Voraussetzungen und Konzepte für die Förderung von Kindern und Jugendlichen mit Migrationshintergrund. Stuttgart: Ernst Klett. S. 251-268. 
Knobbe, Thorsten / Leis, Mario / Umnuß, Karsten (2014): Arbeitszeugnisse. Textbausteine und Tätigkeitsbeschreibungen. Freiburg: Haufe.

Krumm, Hans-Jürgen (2007): Von der Gefährlichkeit der Schlange oder: Textkompetenz im Bildungsdrang von MigrantInnen. In: Schmölzer-Eibinger, Sabine / Weidacher, Georg (Hrsg.): Textkompetenz. Eine Schlüsselkompetenz und ihre Vermittlung. Tübingen: Narr Francke Attempto. S. 199-206.

Maiwald, Klaus (2010): Kompetenzen und Unterrichtsziele im Lese- und Literaturunterricht der Sekundarstufe I. In: Boogaart, Michael Kämper-van den / Spinner, Kaspar H. (Hrsg.): Lese- und Literaturunterricht Teil 2. Handbuch Deutschunterricht in Theorie und Praxis 11/2. Baltmannsweiler: Schneider Hohengehren.

Meier, Jörg (2006): Angewandte Textlinguistik und DaF-Unterricht. In: Scherner, Maximilian / Ziegler, Arne (Hrsg.): Angewandte Textlinguistik. Perspektiven für den Deutsch- und Fremdsprachenunterricht. Tübingen: Narr Francke Attempto. S 193-203.

Portmann-Tselikas, Paul R. (2005): Was ist Textkompetenz? https:/www.uzh.ch/ds/wiki/ssldir/Textkompetenz/uploads/Main/PortmannText-kompetenz.pdf (17.09.2017).

SchmölzerEibinger, Sabine (2011): Lernen in der Zweitsprache. Grundlagen und Verfahren der Förderung von Textkompetenz in mehrsprachigen Klassen. Tübingen: Narr Francke Attempto.

Wöllstein, Angelika / Dudenredaktion (2016): Duden - Die Grammatik. Unentbehrlich für richtiges Deutsch. Der Aufbau der deutschen Sprache vom laut über das Wort und den Satz bis hin zum Text und zu den Merkmalen der gesprochenen Sprache. Berlin u.a.: Dudenverlag.

Zákoník práce (2006): Sbírka zákonů, částka 84, zákon 262 ze dne 21. dubna 2006. https:// www.epravo.czl_dataPublic/sbirky/archiv/sb084-06.pdf (18.3.2018).

Tomáš Káňa

Masarykova univerzita / Universität Wien kana@ped.muni.cz
Justina Kirchner

Universität Wien

justine_kirchner@gmx.at 